\title{
Optic Nerve Tortuosity and Globe Proptosis in Normal and Glaucoma Subjects
}

Xiaofei Wang, $\mathrm{PhD}^{1,2}$, Helmut Rumpel, $\mathrm{PhD}^{3}$, Mani Baskaran, DNB, PhD ${ }^{4,5}$, Tin A Tun, $\mathrm{MD}^{2,4}$, Nicholas Strouthidis, FRCS(Ed), $\mathrm{PhD}^{4,6,7}$, Shamira A Perera, FRCOphth ${ }^{4,5}$, Monisha E Nongpiur, MD, PhD ${ }^{4,5}$, Winston Eng Hoe Lim, MBBS, FRCR ${ }^{3}$, Tin Aung, FRCS(Ed), PhD ${ }^{4,5}$, ${ }^{8}$, Dan Milea, MD, $\mathrm{PhD}^{4,5}$, Michaël J. A. Girard, $\mathrm{PhD}^{2,4}$

1. Beijing Advanced Innovation Center for Biomedical Engineering, School of Biological Science and Medical Engineering, Beihang University, Beijing, China.

2. Ophthalmic Engineering \& Innovation Laboratory, Department of Biomedical Engineering, Faculty of Engineering, National University of Singapore, Singapore

3. Department of Diagnostic Radiology, Singapore General Hospital, Singapore

4. Singapore Eye Research Institute, Singapore National Eye Centre, Singapore

5. Duke-NUS Medical School, Singapore

6. NIHR Biomedical Research Centre, Moorfields Eye Hospital NHS Foundation Trust and UCL Institute of Ophthalmology, London, UK

7. Discipline of Clinical Ophthalmology and Eye Health, University of Sydney, Sydney, NSW, Australia

8. Department of Ophthalmology, Yong Loo Lin School of Medicine, National University of Singapore, Singapore

Meeting Presentation:

Financial Support:

Financial Disclosure:

Conflict of Interest:

Running Head:

Keywords:

Word count:

Figures:

Corresponding Author:
None

Girard acknowledges support from the Singapore Eye Research Institute Pilot Grant (R1228/34/2015) and from an NUS Young Investigator Award (NUSYIA_FY13_P03, R-397-000-174-133).

None

No conflicting relationship exists for any author

Optic nerve tortuosity and globe proptosis in glaucoma

Glaucoma, Eye movements, Optic nerve tortuosity, Globe proptosis

2,845 (Manuscript Text)

3

Dr Michaël J.A. Girard

Ophthalmic Engineering \& Innovation Laboratory

Department of Biomedical Engineering

National University of Singapore

Engineering Block 4, \#04-8

4 Engineering Drive 3

Singapore, 117583

mgirard@nus.edu.sg 


\section{Précis}

Eyes with glaucoma have tauter optic nerves compared with normal eyes, which may exert more force on the optic nerve head tissues during eye movements.

\section{ABSTRACT}

Purpose: To assess the difference in optic nerve tortuosity during eye movements and globe proptosis between primary open angle glaucoma and normal subjects using orbital magnetic resonance imaging.

Methods: 10 Chinese subjects matched for age, ethnicity and refractive errors were recruited, including five normal controls and five patients with primary open angle glaucoma. All subjects underwent magnetic resonance imaging to assess their optic nerves and globes for three eye positions: primary gaze, adduction and abduction. Optic nerve tortuosity (optic nerve length divided by the distance between two ends) and globe proptosis (maximum distance between cornea and interzygomatic line) were measured from magnetic resonance imaging images.

Results: In adduction, the tortuosity of normal eyes was significantly larger than that of the glaucomatous eyes. Optic nerve tortuosity in adduction in the control and glaucoma groups were $1.004 \pm 0.003$ (mean \pm standard deviation) and $1.001 \pm 0.001$, respectively $(p=0.037)$. Globe proptosis (primary gaze) in glaucoma subjects $(19.14 \pm 2.11 \mathrm{~mm})$ was significantly higher than that in control subjects $(15.32 \pm 2.79$ $\mathrm{mm} ; \mathrm{p}=0.046)$

Conclusions: In this sample, subjects with glaucoma exhibited more taut optic nerves and more protruding eye globes compared to normal eyes. This may impact optic nerve head deformations in anatomically predisposed patients. 


\section{INTRODUCTION}

The main site of retinal ganglion cell (RGC) damage in glaucoma is the optic nerve head $(\mathrm{ONH})$, especially the lamina cribrosa $(\mathrm{LC})$ within the $\mathrm{ONH}$. To date, the pathogenesis of glaucoma is not fully elucidated. We know that elevated intraocular pressure (IOP) is the major risk factor for glaucoma. ${ }^{1,2}$ However, glaucoma also occurs in patients with IOP within normal levels, ${ }^{3}$ and may not develop in those with elevated IOP. In short, our understanding of glaucoma is insufficient: While IOP is crucial, it is likely that risk factors other than IOP play an important role in the development and progression of this blinding disease.

The biomechanical theory of glaucoma hypothesizes that elevated IOP deforms the $\mathrm{ONH}$ tissues, and that these deformations (directly or indirectly) drive RGC death. ${ }^{4}$ This theory is supported by much circumstantial evidence ${ }^{5-7}$, and can explain differing sensitivities to IOP. However, IOP is not the only load that can induce ONH deformations. For instance, the cerebrospinal fluid pressure (CSFP) in the optic nerve (ON) subarachnoid space can deform the $\mathrm{ONH}$, and in fact, low CSFP is thought to be linked with the development of normal tension glaucoma. ${ }^{8}$ Recently, eye movements have also been shown to significantly deform the $\mathrm{ON}$ and $\mathrm{ONH}$ tissues. Specifically, recent studies that employed optical coherence tomography (OCT), ${ }^{9-12}$ computational modeling, ${ }^{13-15}$ and magnetic resonance imaging (MRI) ${ }^{16,17}$ all converge to the finding that the optic nerve (ON) can pull the back of the eye during eye movements to result in large deformations within the optic nerve head $(\mathrm{ONH})$ tissues. ${ }^{13,15,16}$

There is now a growing interest to understand whether $\mathrm{ONH}$ deformations induced by the ON traction force could initiate the development and progression of 
optic neuropathies including glaucoma. ${ }^{9}, 11,12,16,18$ Using FE modeling, we have previously predicted that a stiff dura would generate large $\mathrm{ONH}$ deformations. ${ }^{13}$ Moreover, and from a strict mechanical point of view, we have hypothesized that a smaller amount of ON slack in primary gaze (i.e. a less tortuous ON) would intuitively result in larger $\mathrm{ONH}$ deformations during eye movements due to a rapid straightening and pulling effect of the ON. While it is not yet feasible to measure the stiffness of the dura in vivo, ON tortuosity can be assessed with MRI. A recent study has reported that optic nerves of normal tension glaucoma patients are more tortuous than those of normal controls. ${ }^{17}$ However, no studies have investigated the optic nerve morphology in high-tension glaucoma in Chinese subjects.

The aim of this study was to measure ON tortuosity and globe proptosis in healthy and subjects with primary open glaucoma (POAG) in different gaze positions using MRI.

\section{METHODS}

\section{Subject Recruitment \& Clinical Tests}

For this study, ten Chinese subjects were recruited from the Singapore National Eye Center. Among these, five were normal controls and five were diagnosed with primary open angle glaucoma (POAG); 3 were female and 7 were male. Normal controls were recruited from Singapore National Eye Center staff and the general public by advertising. Inclusion criteria for normal subjects were: age $\geq$ 50 years, phakic, and with refractive errors within the range of \pm 3.5 Diopters. POAG cases were diagnosed by the presence of glaucomatous optic neuropathy, defined 
as vertical cup-to-disc ratio of $>0.7$ and/or neuroretinal rim narrowing with an associated visual field defect on standard automated perimetry. Visual field loss for POAG cases should less than $-6 \mathrm{~dB}$ mean deviation value. The glaucoma subjects were newly diagnosed or receiving pharmacologic treatment. We excluded subjects with thyroid eye disease, a history of intraocular surgery, strabismus, ocular motor palsies, orbital or brain tumors, orbital or brain surgeries and subjects that were not suitable for MRI scanning.

All the subjects underwent the following ocular examinations for both eyes: 1) measurement of refraction using an autokeratometer (RK-5, Canon, Tokyo, Japan); 2) measurement of vertical cup-to-disc ratio using a slit lamp. 3) measurement of visual acuity using a logMar chart; 4) anterior segment swept source OCT imaging (SS-1000, CASIA, Tomey, Nagoya, Japan) and 5) measurement of IOP using a Goldmann applanation tonometry (AT900D; Haag-Streit, Köniz, Switzerland). Visual fields were assessed by standard automated perimetry (SITA-FAST 24-2 program; Humphrey Field Analyzer II-750i, Carl Zeiss Meditec, Dublin, CA, USA) for both eyes in glaucoma subjects.

The protocol was approved by the SingHealth Centralized Institutional Review Board and adhered to the ethical principles outlined in the Declaration of Helsinki. Written voluntary informed consent was obtained from each subject.

\section{Magnetic Resonance Imaging}

Each subject's orbital regions were imaged with a 3T MRI scanner and a 32channel head coil (Magnetom Skyra, Siemens Healthcare Sector, Erlangen, Germany) without the use of any contrast agent. FL3D (3D flash sequence) used the following parameters: TR $10 \mathrm{~ms}$, TE $3 \mathrm{~ms}$, flip angle $22^{\circ}$, FOV $140 \mathrm{~mm}$, matrix $256 \mathrm{x}$ 
$256 \times 8$, resulting in the axial images with an in-plane resolution of $0.5 \mathrm{~mm}$ per pixel and a slice thickness of $2 \mathrm{~mm}$. With $60 \%$ oversampling and two averages, the acquisition time for each scan was $1.06 \mathrm{~min}$. The location of the MRI slice was carefully determined during acquisition so that it divided the $\mathrm{ON}$ and the eye globe into superior and inferior halves.

During the MRI procedure, the subjects were orally instructed to direct their gaze on one of the three visual targets, allowing image acquisition in the primary position (baseline), and on left and right gaze (fixed order). The exact eye rotation amplitudes were measured from MRI images in the post-processing stage. These three visual targets were around $32 \mathrm{~cm}$ from subjects' eyes and were 1 inch in width (visual angle of about $4.5^{\circ}$ ) to achieve a very good visibility. After the subjects were positioned in the MRI scanner, they were trained to direct their gaze on individual targets before the MRI session.

\section{Image Delineation}

For each eye, the obtained 2D MRI image was delineated by a single grader (XW) for further analysis using custom-written software in MATLAB (Version 2015a; Mathworks, Inc., Natick, MA, USA). Six structures were delineated manually (Figure 1A): the anterior cornea (in green), the lens capsule and center (yellow), the scleral shell (in red), the temporal and nasal boundaries of the ON (in yellow), and the interzygomatic line (in green). Specifically, for the cornea, $\sim 6$ points were marked that were fitted with a quadratic polynomial. For the lens capsule, $\sim 10$ points were marked that were fitted with an ellipse; the ellipse center was then assumed to be that of the lens. The scleral shell was also identified ( $\sim 30$ discrete points) and fitted with an ellipse to approximate the globe. For the $\mathrm{ON}, \sim 10$ discrete points were 
marked to identify its temporal or nasal boundary, which were then connected using a cubic spline. Finally, two points at the anterior limits of the left and right zygomatic bones were identified and a line that passed through those 2 points was defined as the interzygomatic line. ${ }^{19}$

A central line passing through the $\mathrm{ON}$ was derived from the delineated temporal and nasal ON boundaries, from which we extracted its tortuosity (defined as ON tortuosity).

\section{Measurement of Optic Nerve Tortuosity}

The amount of ON 'slack' is an important factor that may influence the magnitude of the $\mathrm{ON}$ traction force during eye movements. For instance, a smaller amount of slack (i.e. a less tortuous ON) in some individuals may result in larger $\mathrm{ONH}$ deformations during eye movements due to a rapid straightening effect of the ON. To this end, we measured the tortuosity of the ON for each eye. As the posterior end of the intraorbital $\mathrm{ON}$ were faint, tortuosity was calculated based on a $20 \mathrm{~mm}$ long ON segment. ON tortuosity was defined as the length of the ON central line segment ( $20 \mathrm{~mm}$ for all eyes in this study) divided by the distance between two end points of the line segment (minimum path in the axial MRI image). The calculations of length and distance were automatically performed in MATLAB. We reported ON tortuosities for all eyes in all three gaze positions. Note that the ON tortuosity was calculated based on the fitted boundaries of the globe and ON. Therefore, subpixel resolution can be achieved in this measurement.

\section{Measurement of Globe Proptosis on MRI}


We also measured the globe proptosis on axial MRI scans, in baseline gaze position, which was defined as the maximum distance between the cornea and the interzygomatic line. The distance was calculated automatically by our application using the delineated structures (Figure 1A).

\section{Statistical Analysis}

As data from both eyes were used, linear mixed models were employed to adjust for inter-eye correlations. All statistical analyses were performed using $R(R$ Foundation, Vienna, Austria).

\section{RESULTS}

\section{Subjects}

The mean age $( \pm S D)$ for all subjects at the time of MRI scanning was $61.6 \pm 7.6$ years. The mean IOP for POAG eyes before and after IOP lowering treatment were $26.4 \pm 4.6 \mathrm{mmHg}$ and $14.1 \pm 2.3 \mathrm{mmHg}$, respectively. The mean IOP for normal eyes was $15.3 \pm 3.6 \mathrm{mmHg}$. Eye rotation angles were $31 \pm 5^{\circ}$ for abduction and $37 \pm 4^{\circ}$ for abduction as measured from the MRI images.

\section{Axial MRI Images}

Figure 1B shows axial orbital MRI images in a glaucoma patient and in a healthy control, fixating the central, the left and the right visual targets. In adduction, the ONs were highly stretched and were tauter than in normal positions. In abduction, the ONs were also stretched but were more tortuous than those in adduction. 


\section{Optic Nerve Tortuosity}

Normal vs Glaucoma. ON tortuosity in baseline gaze, adduction and abduction of $30^{\circ}$ are summarized in Figure 2 as box plots for both control and glaucoma groups (note that the ON central line was also drawn for each subject to ease interpretation of our results). In each position, the ON tortuosity of normal eyes was significantly larger than that of the glaucomatous eyes. In baseline gaze, ON tortuosity (mean \pm SD) in the control and glaucoma groups was $1.013 \pm 0.019$ and $1.002 \pm 0.003$, respectively $(p=0.248)$; in adduction, $1.004 \pm 0.003$ and $1.001 \pm 0.001$, respectively $(p=0.037)$; in abduction, $1.033 \pm 0.034$ and $1.011 \pm 0.009$, respectively $(p=0.215)$.

Adduction vs Abduction vs Baseline Gaze. For all eyes, ON tortuosity in adduction $(1.003 \pm 0.003)$ was on average smaller than that in baseline gaze $(1.008 \pm 0.014 ; p=0.0423)$. In abduction, ON tortuosity $(1.022 \pm 0.027)$ was significantly larger than that in baseline $(p<0.001)$ and in adduction $(p<0.001)$.

\section{Globe Proptosis}

Proptosis. We found that globe proptosis (baseline gaze) in glaucoma subjects $(19.14 \pm 2.11 \mathrm{~mm})$ was significantly higher than that in control eyes (15.32 $\pm 2.79 \mathrm{~mm} ; \mathrm{p}=0.046$; Figure 3).

\section{DISCUSSION}

Glaucoma is a heterogeneous disease with a pathogenesis that may involve many factors such as age, ethnicity, genes, IOP and cerebrospinal fluid pressure (CSFP) ${ }^{20}$. ONH deformations induced by IOP or CSFP and their correlations with 
glaucoma have been studied extensively ${ }^{21}$. Eye movements can also deform the ONH significantly ${ }^{9,11-13,16}$, but to date, a potential biomechanical link with glaucoma remains unclear. A recent study ${ }^{17}$ has reported that the $\mathrm{ON}$ in patients with normal tension glaucoma are more tortuous than those of controls. However, no studies have investigated the optic nerve morphology in high-tension POAG in Chinese subjects. Our study suggests that ONs in glaucoma subjects are more taut than ONs in normal controls in adduction. The implications of such a finding are still unclear, but a more taut $\mathrm{ON}$ has the potential to result in an earlier extinction of $\mathrm{ON}$ redundancy during eye movements and cause greater stretch within the $\mathrm{ON}$ and $\mathrm{ONH}$. However, ONH deformation levels are also dependent on the biomechanical properties of the dura mater and of the peripapillary sclera ${ }^{13,15}$. Whether a more taut ON would be associated with higher glaucoma susceptibility still remains speculative.

In this study, we found that globe proptosis in normal subjects was $15.32 \pm 2.79 \mathrm{~mm}$, which is highly consistent with data obtained from a large cohort of Chinese adults ${ }^{22}(15.3 \pm 2.3 \mathrm{~mm}$ for patients aged between 70 and 87 years old; 15.7 \pm 1.8 for patients aged between 20 and 69 years old; measured with Hertel Exophthalmometry ${ }^{23}$ ). Interestingly, we also found that globe proptosis in glaucoma subjects (19.14 $\pm 2.11 \mathrm{~mm}$; Figure 3 ) was conspicuously higher than in normal eyes. This difference in proptosis was not caused by elongation of the globe as we found no significant difference in axial length between normal and glaucoma groups (Supplementary material A). We believe this is an interesting but controversial result that would need to be validated in a larger population.

In adduction, ON tortuosity was smaller than that in baseline gaze. This finding is intuitive as the $\mathrm{ON}$ is highly stretched in adduction and thus less curved. On the contrary, ON tortuosity in abduction for both glaucoma and normal groups 
was larger than that in baseline gaze. This result may appear counterintuitive because, in abduction, the $\mathrm{ON}$ is likely to exhibit a certain level of stretch and the distance between the $\mathrm{ONH}$ and the orbital apex is increasing. ${ }^{12}$ This phenomenon could potentially be explained by the combination of: (1) a smaller ON traction force in abduction (as predicted through finite element modeling ${ }^{15}$ ); and (2) resistance from the orbital tissues (fat and extraocular muscles) to restrict the 'free movement' of the $\mathrm{ON}$ in abduction. However, to date, this resistance effect has not been well studied. It is possibly very complex due to nonlinear viscoelastic material properties of the orbital fat, and heterogeneous frictional effects between the orbital fat and the wall of the $\mathrm{ON}^{24}$ Several research groups have suggested that the orbital fat, although soft, is able to provide essential supports for stabilizing the extraocular muscle bellies during eye movements, ${ }^{25,} 26$ while others have suggested that the orbital fat is more like a fluid with no resistance. ${ }^{16}$ Additionally, changes in extraocular muscle shapes and volumes (induced by contraction and relaxation) during eye movements may influence the deformations of surrounding tissues and may subsequently affect $\mathrm{ON}$ deformations. Further studies are needed to investigate the biomechanical properties of the human orbital fat and their interactions with the $\mathrm{ON}$ and extraocular muscles.

The prevalence of high tension glaucoma, normal tension glaucoma and ocular hypertension has been reported to be significantly higher in patients with thyroid eye disease (TED; which may cause globe protrusion) than in the general population. ${ }^{27-30}$ The exact mechanisms of ocular hypertension in TED are not completely understood and were hypothesized to be caused by an increase in episcleral venous pressure induced by elevated retrobulbar pressure ${ }^{31}$ and mucopolysaccharide deposition in the trabecular meshwork. ${ }^{29}$ However, why a 
higher prevalence of normal tension glaucoma is observed in TED cannot yet be explained. Other factors, other than IOP, may be involved. We speculate that prolonged proptosis and/or muscle enlargement could restrict the free motion of the ON during eye movements, which may in turn result in more tension transmitted to the $\mathrm{ONH}$ and induce axonal insult. We also believe that the effects of IOP, CSFP, or optic nerve traction are not necessarily exclusive but complementary. For instance, gaze-induced $\mathrm{ONH}$ deformations in normal tension glaucoma could play a larger role than IOP while in high tension glaucoma the effects of IOP is more prominent. Moreover, the interplay of ONH loads (i.e. IOP, CSFP and ON traction) may also be of importance in our understanding of glaucoma mechanisms. For instance, Sibony has demonstrated that elevated CSFP in papilledema can increase gaze-induced $\mathrm{ONH}$ deformations. ${ }^{11}$ Furthermore, it is also possible that prolonged abnormal stretches of the globe by the ON during eye movements may alter IOP but this has not yet been fully explored, other than in monkeys. ${ }^{32}$ Finally, IOP and CSFP are chronic loads while eye movements are transient in nature, thus the consequences of high ON traction is likely to be different. Our eyes exhibit frequent movements in daily activities (around 170,000 saccades per day ${ }^{33}$ ) and during sleep. The links between frequent transient $\mathrm{ONH}$ deformation during eye movements and corresponding tissue damage and remodeling are yet to be established.

Limitations. In this study, several limitations warrant further discussion. First, only 10 subjects were recruited. While the sample size is small, all patients were matched for age, ethnicity, and refractive errors to limit the influence of these cofounding factors.

Second, only one 2D axial image was analyzed, and we were not able to capture the potential curvatures of the $\mathrm{ON}$ in the superior-inferior direction. However, 
our preliminary study using 20 young subjects (unpublished data) showed that horizontal eye movement could only cause changes of ON tortuosity in the horizontal plane. Therefore, a 2D MRI image is capable to capture the feature of ON tortuosity during horizontal eye movement. Further studies may use high spatial resolution images to study the 3D shape changes of ON during eye movements.

Third, the length of the ON was not measured in this study because we were not able to identify the optic canal precisely from our MRI scans. Although an estimate is possible using the medial rectus muscle end, the values may not be reliable. The intraorbital ON length may be a very important factor that influences ON stretching during eye movement. Future studies should include this important parameter whenever possible.

\section{CONCLUSIONS}

In conclusion, we demonstrated a more taut $\mathrm{ON}$ and a more protruding globe in glaucoma subjects in a small population, which may result in larger deformations of the ONH during eye movements. 


\section{FIGURES}
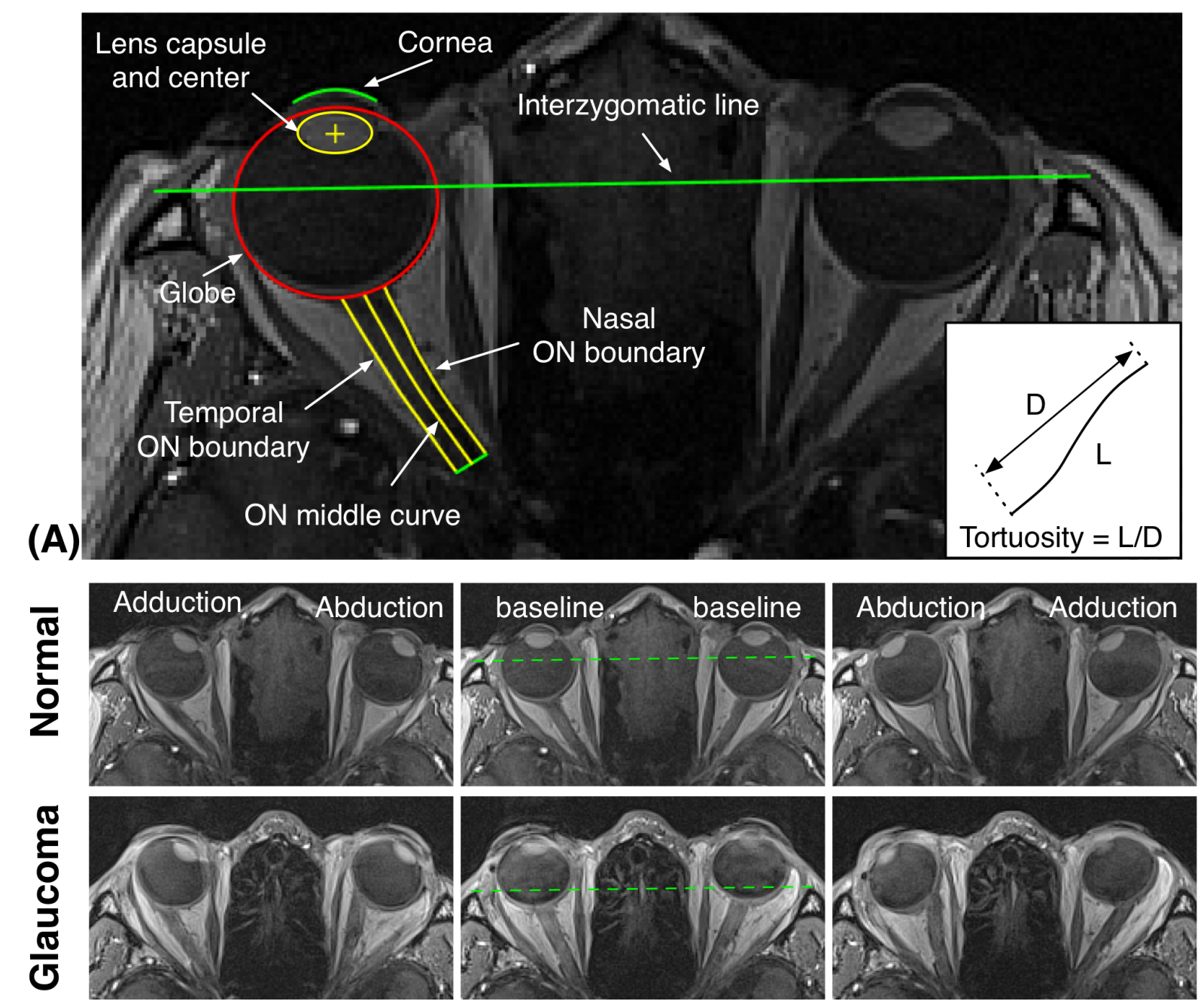

(B)

Left gaze

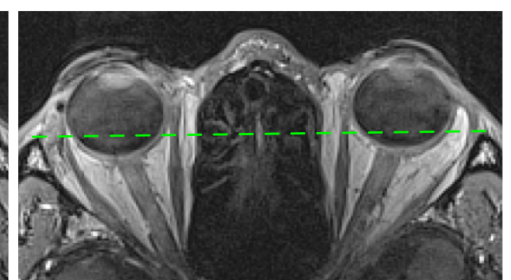

Primary gaze

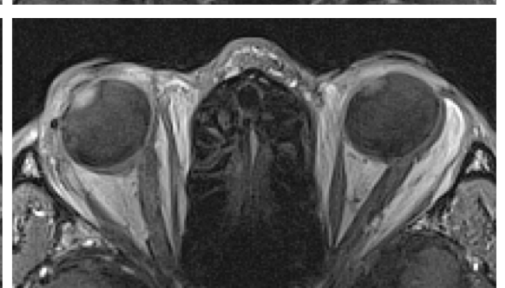

Right gaze

Figure 1. (A) Delineated features of the eye and the orbit. All measurements were calculated automatically using our custom-written application based on these features. Figure on the right corner indicates tortuosity definition. (B) Axial MRI images for a glaucoma and a normal subject in the primary, left and right gaze positions. Green dashed lines indicate interzygomatic lines. Note the high proptosis in the glaucoma subject. 


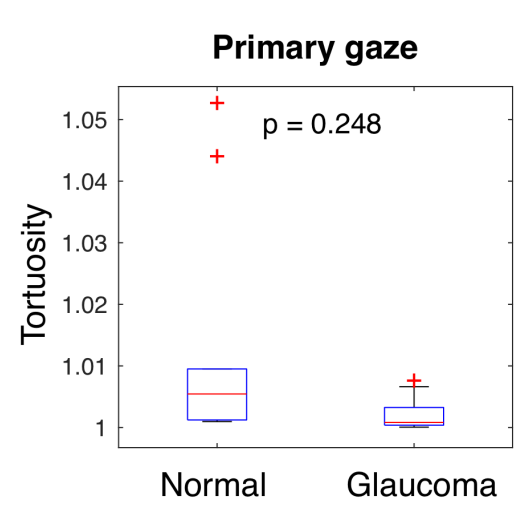

\section{Tortuosity}
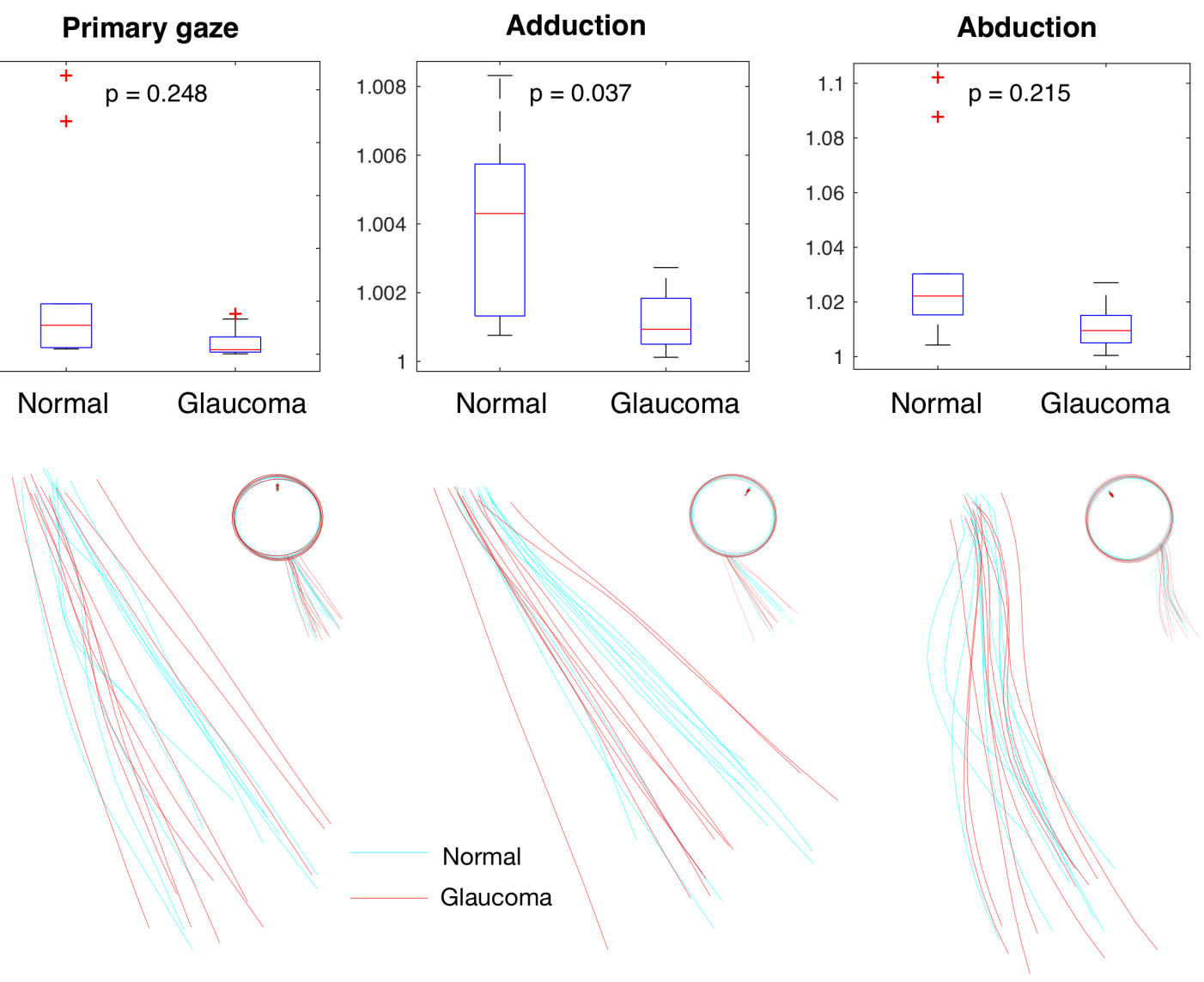

Figure 2. Upper row: box plots of $\mathrm{ON}$ tortuosities for normal and glaucoma subjects in primary gaze, adduction and abduction. ON tortuosities of normal eyes in adduction were statistically higher than those of glaucoma subjects. Lower row: delineated ON midlines at various eye positions. 


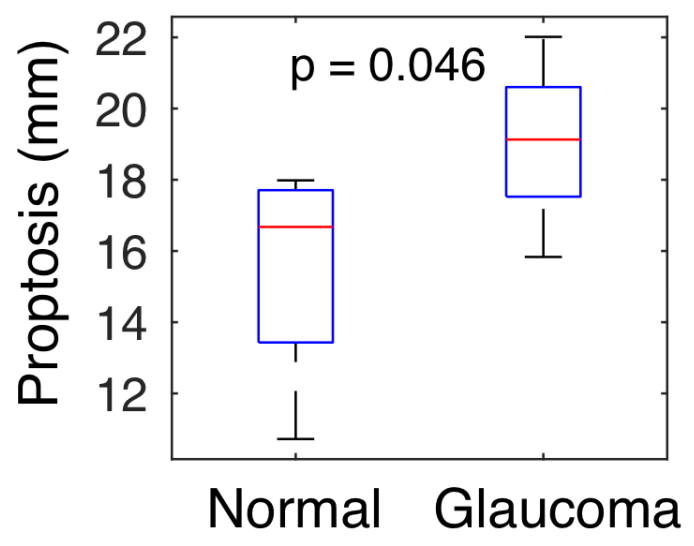

Figure 3. Box plots of globe proptosis for normal and glaucoma subjects in primary gaze. Proptoses of glaucomatous eyes were higher than those of the normal eyes. 


\section{REFERENCES}

1. Sommer A, Tielsch JM, Katz J, et al. Relationship between intraocular pressure and primary open angle glaucoma among white and black Americans. The Baltimore Eye Survey. Arch Ophthalmol 1991;109:1090-1095.

2. Leske MC, Wu SY, Hennis A, et al. Risk factors for incident open-angle glaucoma: the Barbados Eye Studies. Ophthalmology 2008;115:85-93.

3. Leske MC. Open-angle glaucoma - An epidemiologic overview. Ophthal Epidemiol 2007;14:166-172.

4. Burgoyne CF, Downs JC, Bellezza AJ, et al. The optic nerve head as a biomechanical structure: a new paradigm for understanding the role of IOP-related stress and strain in the pathophysiology of glaucomatous optic nerve head damage. Prog Retin Eye Res 2005;24:3973.

5. Sigal IA, Flanagan JG, Tertinegg I, et al. Predicted extension, compression and shearing of optic nerve head tissues. Exp Eye Res 2007;85:312-322.

6. Kirwan RP, Crean JK, Fenerty $\mathrm{CH}$, et al. Effect of cyclical mechanical stretch and exogenous transforming growth factor-beta1 on matrix metalloproteinase-2 activity in lamina cribrosa cells from the human optic nerve head. J Glaucoma 2004;13:327.

7. Quigley HA, Addicks EM. Regional differences in the structure of the lamina cribrosa and their relation to glaucomatous optic nerve damage. Arch Ophthalmol-Chic 1981;99:137.

8. Berdahl JP, Fautsch MP, Stinnett SS, et al. Intracranial Pressure in Primary Open Angle Glaucoma, Normal Tension Glaucoma, and Ocular Hypertension: A Case-Control Study. Invest Ophthalmol Vis Sci 2008;49:5412-5418.

9. Chang MY, Shin A, Park J, et al. Deformation of Optic Nerve Head and Peripapillary Tissues By Horizontal Duction. American Journal of Ophthalmology 2017;174:85-94.

10. Suh SY, Le A, Shin A, et al. Progressive Deformation of the Optic Nerve Head and Peripapillary Structures by Graded Horizontal Duction. Invest Ophthalmol Vis Sci 2017;58:5015-5021.

11. Sibony PA. Gaze Evoked Deformations of the Peripapillary Retina in Papilledema and Ischemic Optic Neuropathy. Invest Ophthalmol Vis Sci 2016;57:4979-4987.

12. Wang X, Beotra MR, Tun TA, et al. In Vivo 3-Dimensional Strain Mapping Confirms Large Optic Nerve Head Deformations Following Horizontal Eye Movements. Invest Ophthalmol Vis Sci 2016;57:5825-5833.

13. Wang $X$, Rumpel H, Lim WEH, et al. Finite Element Analysis Predicts Large Optic Nerve Head Strains During Horizontal Eye Movements. Invest Ophthalmol Vis Sci 2016;57:2452-2462.

14. Wang X, Rumpel H, Lim WEH, et al. Author Response: Peripapillary Suprachoroidal Cavitation, Parapapillary Gamma Zone and Optic Disc Rotation due to the Biomechanics of the Optic Nerve Dura Mater. Invest Ophthalmol Vis Sci 2016;57:4374-4375.

15. Wang X, Fisher LK, Milea D, et al. Predictions of Optic Nerve Traction Forces and Peripapillary Tissue Stresses Following Horizontal Eye Movements. Invest Ophthalmol Vis Sci 2017;58:2044-2053.

16. Demer JL. Optic Nerve Sheath as a Novel Mechanical Load on the Globe in Ocular Duction. Invest Ophthalmol Vis Sci 2016;57:1826-1838. 
17. Demer JL, Clark RA, Suh SY, et al. Magnetic Resonance Imaging of Optic Nerve Traction During Adduction in Primary Open-Angle Glaucoma With Normal Intraocular Pressure. Invest Ophthalmol Vis Sci 2017;58:4114-4125.

18. Shin YU, Lim HW, Kim JH. Changes of optic nerve head induced by eye movement. Neurology 2016.

19. Lee JS, Lim DW, Lee SH, Oum BS, et al. Normative measurements of Korean orbital structures revealed by computerized tomography. Acta Ophthalmol Scan 2001;79:197-200. 20. Doucette LP, Rasnitsyn A, Seifi M, et al. The interactions of genes, age, and environment in glaucoma pathogenesis. Surv Ophthalmol 2015;60:310-326.

21. Jonas JB, Wang N, Yang D, et al. Facts and myths of cerebrospinal fluid pressure for the physiology of the eye. Prog Retin Eye Res 2015;46:67-83.

22. Wu D, Liu X, Wu D, et al. Normal Values of Hertel Exophthalmometry in a Chinese Han Population from Shenyang, Northeast China. Sci Rep-Uk 2015;5:8526.

23. Ramli N, Kala S, Samsudin A, et al. Proptosis--Correlation and Agreement between Hertel Exophthalmometry and Computed Tomography. Orbit 2015;34:257-262.

24. Hotte GJ, Schaafsma PJ, Botha CP, et al. Visualization of Sliding and Deformation of Orbital Fat During Eye Rotation. Trans/ Vis Sci Techn 2016;5:9.

25. Schoemaker I, Hoefnagel PPW, Mastenbroek TJ, et al. Elasticity, viscosity, and deformation of orbital fat. Investigative Ophthalmology \& Visual Science 2006;47:4819-4826.

26. Schutte $S$, van den Bedem SP, van Keulen F, et al. A finite-element analysis model of orbital biomechanics. Vision Res 2006;46:1724-1731.

27. Cockerham KP, Pal C, Jani B, et al. The prevalence and implications of ocular hypertension and glaucoma in thyroid-associated orbitopathy. Ophthalmology 1997;104:914-917.

28. Ohtsuka K, Nakamura Y. Open-angle glaucoma associated with graves disease. Am J Ophthalmol 2000;129:613-617.

29. Lee AJ, Rochtchina E, Wang JJ, et al. Open-angle glaucoma and systemic thyroid disease in an older population: The Blue Mountains Eye Study. Eye 2004;18:600-608.

30. Cross JM, Girkin CA, Owsley C, et al. The association between thyroid problems and glaucoma. Br J Ophthalmol 2008;92:1503-1505.

31. Ohtsuka K. Intraocular Pressure and Proptosis in 95 Patients with Graves Ophthalmopathy. Am J Ophthalmol 1997;124:570-572.

32. Downs JC, Burgoyne CF, Seigfreid WP, et al. 24-hour IOP telemetry in the nonhuman primate: implant system performance and initial characterization of IOP at multiple timescales. Invest Ophthalmol Vis Sci 2011;52:7365-7375.

33. Schiller PH, Tehovnik EJ. Neural mechanisms underlying target selection with saccadic eye movements. Cortical Function: A View from the Thalamus 2005;149:157-171. 


\section{Supplementary material A}

Axial lengths were calculated based on delineated features of the globe. Specifically, an axial line was drawn through the globe center and the lens center. The distance between the intersections of the axial line with the cornea and the posterior globe was defined as the axial length. The measured axial length for glaucoma and normal eyes was $25.68 \pm 1.11$ and $25.13 \pm 0.77 \mathrm{~mm}$, respectively. There was no statistical difference in axial length between normal and glaucomatous eyes $(p=0.31)$ 
bioRxiv preprint doi: https://doi.org/10.1101/397570; this version posted August 22, 2018. The copyright holder for this preprint (which was

not certified by peer review) is the author/funder, who has granted bioRxiv a license to display the preprint in perpetuity. It is made available under aCC-BY-NC-ND 4.0 International license. 